\title{
Microbial Interactions in the Yoghurt Consortium: Current Status and Product Implications
}

\author{
Sander Sieuwerts* \\ Research Scientist, Arla Foods Strategic Innovation Centre, Rørdrumvej 2, 8220 Brabrand, Denmark
}

Received: May 25, 2016; Accepted: July 11, 2016; Published: July 14, 2016

*Corresponding author: Sander Sieuwerts, Research Scientist, Arla Foods Strategic Innovation Centre, Rørdrumvej 2, 8220 Brabrand, Denmark, Tel: +4587466703; Fax: +4586281691; E-mail: sander.sieuwerts@arlafoods.com

\begin{abstract}
The metabolic potential of the fermenting microbial culture influences the characteristics of fermented products, but the extent to which this occurs depends on environmental factors and the microorganism's interactions with its environment. It is therefore not possible to simply translate gene content to product characteristics and this is particularly the case when cultures are consortia of microorganisms influencing each other. The yoghurt consortium, consisting of Streptococcus thermophilus and Lactobacillus delbrueckii subsp. bulgaricus, is mainly characterized by mutualistic interactions between the two species. This review provides an updated overview of these interactions, based on the latest insights, and their impact on fermentation and product characteristics. Finally, some leads are given on how to use modulation of interactions as means to alter product characteristics.
\end{abstract}

Keywords: Streptococcus thermophilus; Lactobacillus delbrueckii subsp. bulgaricus; Mutualism; Yoghurt; Proteolysis; Exopolysaccharides

\section{Microbial interactions in food fermentations}

Fermentation is a process in which a carbon source is dissimilated by microorganisms yielding energy and without net oxidation. The major end products of this fermentation by microorganisms are generally alcohols and organic acids, such as lactic acid, acetic acid and propionic acid. Many food products are fermented or contain a component of fermentation in the production process. Food is fermented for many reasons. These reasons include improved microbial stability, resulting in shelflife extension and higher safety, improved sensoric properties, and increased availability of essential nutrients either as result of production by the microorganisms or by improved digestibility of the raw material [1]. The metabolic potential of the fermenting microbial culture will thus have a huge impact on the exact characteristics of the fermented product. This metabolic potential is captured in the microorganism's genes, but to what extent the metabolism affects the product properties very much depends on environmental factors such as temperature, micronutrient availability, oxygen pressure and pH and on the microorganism's interactions with its environment. It is therefore not possible to simply translate gene content to product characteristics.
Moreover, by far most cultures applied in food fermentations are microbial consortia consisting of multiple species and/or strains, where the consortium members affect each other by various modes of interaction. The intermicrobial interactions may be (i) direct communication via signaling molecules, (ii) labor division by growth factor exchange, or (iii) effects induced by changed physico-chemical properties of the environment or production of inhibiting compounds [2,3]. These interactions can have a positive, neutral, or negative influence on both the effector and the target, making it possible to classify them based on their mutually beneficial and detrimental effects on fitness [4]. There are six main classes: neutralism, amensalism, competition, commensalism, parasitism and mutualism (Table 1). It must be noted that in a competitive and mutualistic relationship both microorganisms are effector and target at the same time, where in the others there typically is one effector and one target.

\section{Mutualism in the yoghurt consortium}

Yoghurt is bovine milk typically fermented with a combination of the two lactic acid bacteria species Streptococcus thermophilus and Lactobacillus delbrueckii subsp. bulgaricus. Although there are reported cases of competition and amensalism for some strain combinations [5,6], the latter mainly as a result of bacteriocin production, this consortium is typically characterized as one with mutualistic interactions (Figure 1). The mutualistic nature of the below-explained interactions is the basis behind the stability of the yoghurt consortium [7]. Where negative interactions such as competition tend to lead to exclusion of one of the consortium members, mutual dependency or stimulation generally leads to equilibrium. It has been known for a long time that the yoghurt bacteria stimulate

Table 1: The six main classes of microbial interactions based on their beneficial and detrimental effects on fitness of the effector and target microorganisms.

\begin{tabular}{|l|l|l|l|}
\hline \multirow{2}{*}{ Effect on target } & \multicolumn{3}{|c|}{ Effect on effector } \\
\cline { 2 - 4 } & Beneficial & Detrimental & Neutral \\
\hline Beneficial & Mutualism & & Commensalism \\
\hline Detrimental & Parasitism & Competition & Amensalism \\
\hline Neutral & & & Neutralism \\
\hline
\end{tabular}


each other's growth by the exchange of metabolites, a process called protocooperation, but the presence and extent of these interactions depends on the exact combination of strains as there is strain to strain variation in metabolic potential. Typically, the yoghurt fermentation contains two exponential growth phases separated by a transition phase with lower growth [8] (Figure 2 ). The first exponential phase is characterized by growth of $S$. thermophilus, which is more tolerant to neutral $\mathrm{pH}$ and more effective in taking up Amino Acids (AA) and trace elements than L. bulgaricus. During its growth, formic acid and folic acid are produced. This can help purine biosynthesis in L. bulgaricus as precursor and co-factor, respectively, because this bacterium with its highly degraded genome [9] is missing genes for de novo folic acid biosynthesis and is therewith impaired in effective purine biosynthesis. Moreover, $S$. thermophilus consumes oxygen and produces carbon dioxide, thereby benefitting the less oxygen tolerant L. bulgaricus [10]. In the transition phase, growth of $S$. thermophilus slows down, mainly due to a lack of AA, notably sulfur and branched-chain AA, as the free levels of these AA in milk are low and most $S$. thermophilus strains do not express an exoprotease to harvest oligopeptides and AA from milk proteins. During this transition phase, growth of $L$. bulgaricus and its expression of the protease gene prtB are initiated, increasing the levels of oligopeptides that can be taken up by and support a second exponential growth phase of $S$. thermophilus while also supporting exponential growth of L. bulgaricus [8]. The cell-wall resident PrtB, however, does not release sufficient sulfur AA and branched-chain AA as these are only present in minor fractions of casein compared to the microorganisms' requirements.
Therefore genes in the pathways for these AA are the only de novo AA biosynthesis genes that are upregulated during this second exponential growth phase [8,11]. Other genes typically upregulated in $S$. thermophilus during this phase are involved in long-chain fatty acids production. L. bulgaricus, having an incomplete pathway for de novo biosynthesis of these compounds, may benefit of this. In addition, gene expression of pathways for production of Exopolysaccharides (EPS) is elevated, as evidenced by the increasing amount of EPS that is present in the system, which continues in the stationary phase. This increased expression of EPS genes may be a direct result of the increasing availability of nitrogen source, i.e. casein hydrolysis products $[12,13]$. It is hypothesized that EPS here play a role in ensuring close proximities between the two species, thereby facilitating the exchange of metabolites [14], additional to protection against unfavorable conditions, such as high acidity. Cell aggregation, cell to cell communication, protection against environmental factors and accumulation of metal ions are all reported as functions of EPS [15]. A recent study also links $S$. thermophilus urease activity to increased growth of L. bulgaricus [16]. Not only the relation of this reaction - from urea to ammonia and carbamate - with aspartate, glutamine, and arginine and for the interaction relevant carbon dioxide metabolism provides a benefit, but also the released ammonia increases the pH both outside and inside cells, allowing a higher growth and acid production. This deacidification effect only occurs locally, indicating the importance of small distance between the two bacteria. Another recently discovered metabolite exchange involves glutathione, a widely distributed antioxidant that can be produced by $S$. thermophilus but not $L$.

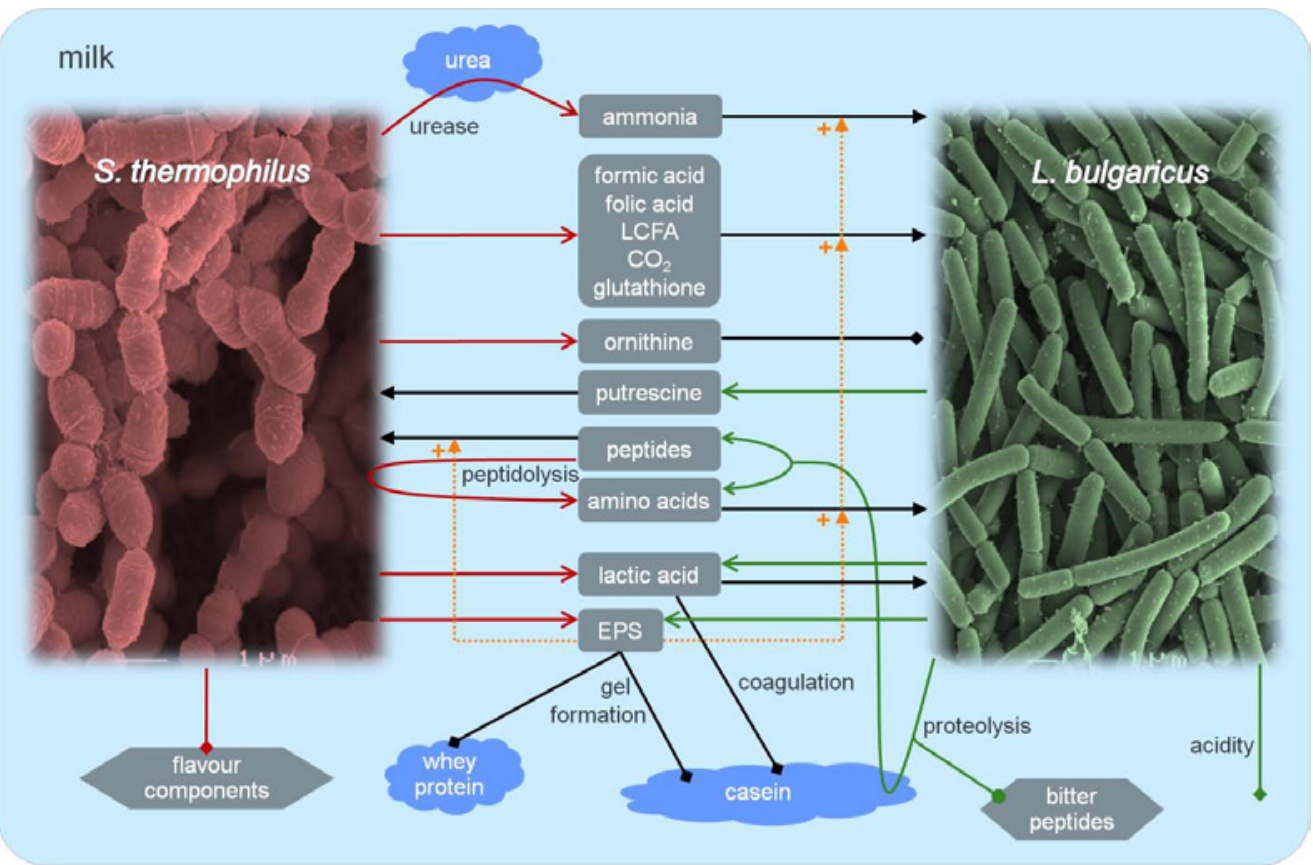

Figure 1: representation of the interactions between S. thermophilus and L. bulgaricus during yoghurt fermentation and their effects on product characteristics. The dotted lines indicate that EPS is hypothesized to facilitate the exchange of metabolites by establishing close proximities between the two species $\rightarrow$ : production or enzymatic activity; $\rightarrow$ : positive effect of the component; $\longrightarrow$ : negative effect; $\longrightarrow$ : neutral or yet to be confirmed effect; EPS: Exopolysaccharides; LCFA: Long-Chain Fatty Acids. 


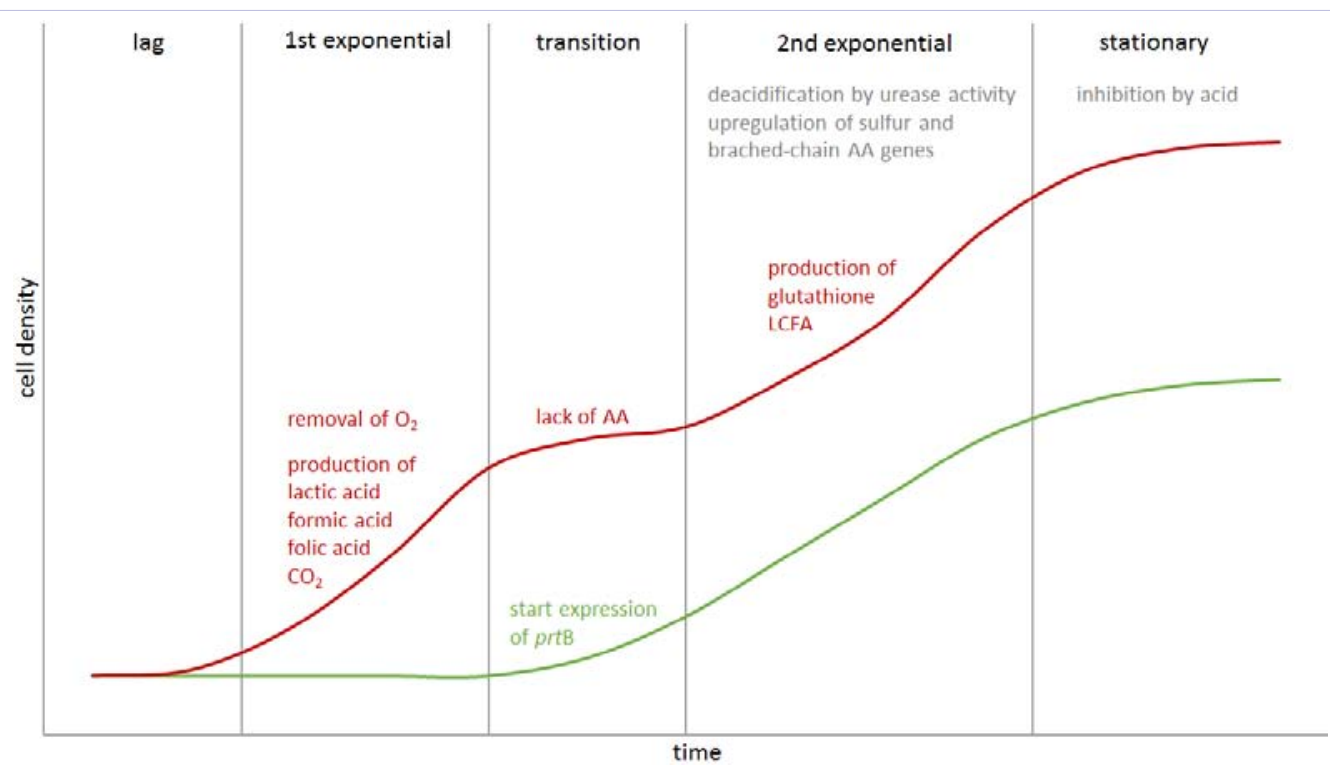

Figure 2: Schematic representation of the growth phases in a typical co-fermentation of S. thermophilus (red) and L. bulgaricus (green) and the most important factors that determine their growth behaviors. AA: Amino Acids; LCFA: Long-Chain Fatty Acids.

bulgaricus, which was shown to relieve acid stress in the latter, resulting in improved growth [17]. Finally, there has also been reported an exchange of ornithine and putrescine between the two species, but the function of this exchange is not elucidated yet. Possible functions include the production of carbon dioxide and the use of putrescine as co-factor in cell division or as metal ion chelation agent [14]. Hereby, it is noteworthy that metal ions, in particular iron and manganese, are quite scarce in milk and efficient systems to take up these ions may certainly benefit growth of the microorganisms. That matches with reported elevated expression of genes involved in iron chelation [11] in $S$. thermophilus in co-culture with L. bulgaricus compared to its mono culture.

\section{Effects of interactions and strains on product characteristics}

The extent and nature of the mutual interactions between the yoghurt bacteria will largely determine the performances of both species and this depends on the exact combinations of strains, moving the equilibrium in the consortium more towards one of the species rather than a one to one ratio. Combinations in which the interactions between the two species are fine-tuned promote the most efficient mutual growth with the least loss or lack of exchanged metabolites. Poor interactions lead to reduced fermentation rate or unbalanced growth as a result of lacking nutrients as can be concluded from combining genome-scale metabolic models of both species [18] or kinetic models [19]. For example, some strains of $S$. thermophilus express the exoprotease PrtS, which makes them independent of L. bulgaricus for their AA supply, even though it has been shown that PrtS has no influence on acidification and microbial composition when PrtB is present. In contrast, when combined with a non-proteolytic $L$. bulgaricus strain, PrtS mainly supports growth of $S$. thermophilus, leading to low counts of L. bulgaricus [20] and altered secondary metabolite accumulation [21]. What are the consequences of these interactions on product characteristics? Affecting the interactions will always result in a shift in microbial composition and therewith impact species-specific contributions to the environment. In the here mentioned example with reduced $L$. bulgaricus counts, the yoghurt is more likely to be mild, because L. bulgaricus is typically associated with lower $\mathrm{pH}$ as this species is generally more acid tolerant than its counterpart. Moreover, since $S$. thermophilus mostly is the largest contributor of EPS and aroma compounds [22], notably of acetoin and diacetyl that together with acetaldehyde make the typical yoghurt flavor, a change in composition in the yoghurt culture will affect viscosity and flavor, as was exemplified in a recent study [21]. In a typical yoghurt culture with a non-proteolytic $S$. thermophilus, high levels of $L$. bulgaricus are not only associated with high acidity but also with bitterness, as the activity of its exoprotease may result in accumulation of bitter tasting peptides and amino acids $[23,24]$. With the current trend in the dairy industry towards milder yoghurts with a good mouthfeel (high viscosity), it is not unsurprising that culture producers try to engineer combinations of $S$. thermophilus and L. bulgaricus strains that result in high counts of the former and low counts of the latter, i.e. cultures in which the interactions between the two species work in such a way that $S$. thermophilus sufficiently benefits from L. bulgaricus' proteolysis while not very much promoting its growth. Using only $S$. thermophilus is often not possible, because proteolysis is required for optimal growth and acidification and because in many countries it is obligatory to have both species, or at least both cocci and bacilli, present and alive in order to be able to call the product yoghurt [25]. Moreover, an effective fermentation with both species and concomitant proteolytic and peptidolytic action is for example essential to decrease allergic reactivity to $\beta$-lactoglobulin and $\alpha$-lactalbumin among $>5 \%$ of the infant population and around $2 \%$ of the adults, as was showcased by 
$\mathrm{Bu}$ and coworkers [26]. The extent of dissimilation of allergic reaction causing proteins and reduction in allergic reactions are, however, highly dependent on the used strains and their proteolytic and peptidolytic capabilities $[27,28]$. Considering such properties of effective fermentations are not in focus of culture producers and dairy industry yet except in few cases where specialized products are made, but with the rise in welfare diseases, healthy and nutritious foods become more and more important.

There are multiple ways to change product characteristics by modifying interactions with the environment and with consortium members. As mentioned, by changing the strain combination, it is possible to acquire different extents of the aforementioned mutualistic interactions resulting in different employments of the microorganisms' metabolic potentials thereby changing the metabolite profile of the yoghurt [21]. This will result in a different taste and texture. As another example, extension of the fermentation time, notably of the second exponential phase, by lowering the fermentation temperature will lead to a final higher production of aroma compounds and EPS [12]. It is not possible to simply assume that higher EPS levels naturally lead to higher viscosity, though. The exact nature of EPS - e.g. capsular or free, electric charge, monomer composition, extent of branching and types of side-groups - together with the amount and composition of milk proteins largely determine viscoelastic and structural properties of the EPS-protein matrix and therewith organoleptic properties such as creaminess and mouth thickness, as was exemplified in two recent studies $[29,30]$. EPS produced by some strains interact better with caseins and others with whey proteins. It is therefore essential for starter culture producers and yoghurt manufacturers to not only pick the right strain combination for an optimal fermentation and flavor balance [21], but also to accommodate the right strain combination with the right protein composition and to use the right fermentation conditions in order to make yoghurt with exactly the desired properties.

\section{References}

1. Smid EJ, Lacroix C. Microbe-microbe interactions in mixed culture food fermentations. Curr Opin Biotechnol. 2013;24(2):148-54. doi: 10.1016/j.copbio.2012.11.007

2. Bull AT, Slater JH. Microbial Interactions and Communities. London: Academic Press; 1982.

3. Taga ME, Bassler BL. Chemical communication among bacteria. Proc Natl Acad Sci U S A. 2003;100 Suppl 2:14549-54.

4. Sieuwerts S, de Bok FA, Hugenholtz J, van Hylckama Vlieg JE Unraveling microbial interactions in food fermentations: from classical to genomics approaches. Appl Environ Microbiol. 2008;74(16):49975007. doi: 10.1128/AEM.00113-08.

5. Moon NJ, Reinbold GW. Commensalism and competition in mixed cultures of Lactobacillus bulgaricus and Streptococcus thermophilus. J Milk Food Technol. 1976;39:337-41.

6. Pereira Martins JF, Luchese RH. The assessment of growth compatibility between strains of Lactobacillus bulgaricus and Streptococcus thermophilus. Rev Inst Lactic Cândido Tostes (Brasil). 1988;43:11-3.
7. Ghosh S, Chowdhury R, Bhattacharya P. Mixed consortia in bioprocesses: role of microbial interactions. Appl Microbiol Biotechnol. 2016;100(10):4283-95. doi: 10.1007/s00253-016-7448-1.

8. Sieuwerts S, Molenaar D, van Hijum SA, Beerthuyzen M, Stevens MJ, Janssen PW, et al. Mixed-culture transcriptome analysis reveals the molecular basis of mixed-culture growth in Streptococcus thermophilus and Lactobacillus bulgaricus. Appl Environ Microbiol. 2010;76(23):7775-84. doi: 10.1128/AEM.01122-10.

9. Van de Guchte M, Penaud S, Grimaldi C, Barbe V, Bryson K, Nicolas P, et al. The complete genome sequence of Lactobacillus bulgaricus reveals extensive and ongoing reductive evolution. Proc Natl Acad Sci U S A. 2006;103(24):9274-9.

10. Sasaki Y, Horiuchi H, Kawashima H, Mukai T, Yamamoto Y. NADH Oxidase of Streptococcus thermophilus 1131 is Required for the Effective Yogurt Fermentation with Lactobacillus delbrueckii subsp. bulgaricus 2038. Biosci Microbiota Food Health. 2014;33(1):31-40. doi: $10.12938 / \mathrm{bmfh} .33 .31$.

11. Herve-Jimenez L, Guillouard I, Guedon E, Boudebbouze S, Hols P, Monnet V, et al. Postgenomic analysis of streptococcus thermophilus cocultivated in milk with Lactobacillus delbrueckii subsp. bulgaricus: involvement of nitrogen, purine, and iron metabolism. Appl Environ Microbiol. 2009;75(7):2062-73. doi: 10.1128/AEM.01984-08.

12. Cerning J. Production of exopolysaccharides by lactic acid bacteria and dairy propionibacteria. Le Lait. 1995;75(4-5):463-72.

13. Zhang Q, Yang B, Brashears MM, Yu Z, Zhao M, Liu N, et al. Influence of casein hydrolysates on exopolysaccharide synthesis by Streptococcus thermophilus and Lactobacillus delbrueckii ssp. bulgaricus. J Sci Food Agric. 2014;94(7):1366-72. doi: 10.1002/jsfa.6420.

14. Sieuwerts S. Analysis of molecular interactions between yoghurt bacteria by an integrated genomics approach. PhD dissertation. Wageningen University. 2009.

15.Zannini E, Waters DM, Coffey A, Arendt EK. Production, properties, and industrial food application of lactic acid bacteria-derived exopolysaccharides. Appl Microbiol Biotechnol. 2016;100(3):112135. doi: $10.1007 / \mathrm{s} 00253-015-7172-2$.

16. Arioli S, Della Scala G, Remagni MC, Stuknyte M, Colombo S, Guglielmetti S, et al. Streptococcus thermophilus urease activity boosts Lactobacillus delbrueckii subsp. bulgaricus homolactic fermentation. Int J Food Microbiol. 2016. pii: S0168-1605(16)30007-1. doi: 10.1016/j.ijfoodmicro.2016.01.006.

17.Wang T, Xu Z, Lu S, Xin M, Kong J. Effects of glutathione on acid stress resistance and symbiosis between Streptococcus thermophilus and Lactobacillus delbrueckii subsp. bulgaricus. International Dairy Journal. 2016;61:22-8. doi: 10.1016/j.idairyj.2016.03.012.

18. Hanemaaijer M, Röling WF, Olivier BG, Khandelwal RA, Teusink B, Bruggeman FJ. Systems modeling approaches for microbial community studies: From metagenomics to inference of the community structure. Front Microbiol. 2015;6:213. doi: 10.3389/fmicb.2015.00213.

19.Aghababaie M, Khanahmadi M, Beheshti M. Developing a kinetic model for co-culture of yogurt starter bacteria growth in $\mathrm{pH}$ controlled batch fermentation. Journal of Food Engineering. 2015;166:72-9. doi: 10.1016/j.jfoodeng.2015.05.013.

20. Courtin P, Monnet V, Rul F. Cell-wall proteinases PrtS and PrtB have a different role in Streptococcus thermophilus/Lactobacillus bulgaricus mixed cultures in milk. Microbiology. 2002;148(Pt 11):3413-21.

21. Settachaimongkon S, Nout MJ, Antunes Fernandes EC, Hettinga KA, Vervoort JM, van Hooijdonk TC, et al. Influence of different 
proteolytic strains of Streptococcus thermophilus in co-culture with Lactobacillus delbrueckii subsp. bulgaricus on the metabolite profile of set-yoghurt. Int J Food Microbiol. 2014;177:29-36. doi: 10.1016/j. ijfoodmicro.2014.02.008

22. Pastink MI, Sieuwerts S, de Bok FAM, Janssen PWM, Teusink B, van Hylckama Vlieg JET, et al. Genomics and high-throughput screening approaches for optimal flavour production in dairy fermentation. International Dairy Journal. 2008;18(8):781-9. doi: 10.1016/j. idairyj.2007.07.006.

23. Roudot Algaron F. The taste of amino acids, peptides and proteins: Examples of tasty peptides in casein hydrolysates. Lait 1996;76(4):313-48.

24. Habibi-Najafi MB, Lee BH. Bitterness in Cheese: A Review. Crit Rev Food Sci Nutr. 1996;36(5):397-411.

25. Tamime AY. Fermented milks: a historical food with modern applications - a review. Eur J Clin Nutr. 2002;56 Suppl 4:S2-S15.

26. Bu G, Luo Y, Zhang Y, Chen F. Effects of fermentation by lactic acid bacteria on the antigenicity of bovine whey proteins. J Sci Food Agric. 2010;90(12):2015-20. doi: 10.1002/jsfa.4046.

27. Tzvetkova I, Dalgalarrondo M, Danova S, Iliev I, Ivanova I, Chobert J-M, et al. Hydrolysis of major dairy proteins by lactic acid bacteria from Bulgarian yogurts. Journal of Food Biochemistry. 2007;31(5):680702. doi: 10.1111/j.1745-4514.2007.00137.x.

28. Jedrychowski L, Wroblewska B. Reduction of the antigenicity of whey proteins by lactic acid fermentation. Food and Agricultural Immunology. 1999;11(1):91-9. doi: 10.1080/09540109999951.

29. Buldo P, Benfeld C, Folkenberg DM, Jensen HB, Amigo JM, Sieuwerts $S$, et al. The role of exopolysaccharide-producing cultures and whey protein ingredients in yoghurt. LWT - Food Science and Technology. 2016;72:189-98. doi: 10.1016/j.lwt.2016.04.050.

30. Buldo P, Benfeldt C, Carey JP, Folkenberg DM, Jensen HB, Sieuwerts S, et al. Interactions of milk proteins with low and high acyl gellan: Effect on microstructure and textural properties of acidified milk. Food Hydrocolloids. 2016;60:225-31. doi: 10.1016/j.foodhyd.2016.03.041. 